\title{
Touch the 3rd Dimension! Understanding Stereoscopic 3D Touchscreen Interaction
}

\author{
Ashley Colley ${ }^{1}$, Jonna Häkkila ${ }^{1}$, Johannes Schöning ${ }^{2}$, Florian Daiber ${ }^{3}$, Frank \\ Steinicke ${ }^{4}$, Antonio Krüger ${ }^{3}$ \\ ${ }^{1}$ CIE, University of Oulu, Oulu, Finland \\ ashley.colley@cie.fi, jonna.hakkila@cie.fi \\ ${ }^{2}$ Hasselt University, tUL - iMinds, Diepenbeek, Belgium \\ johannes.schoening@uhasselt.be \\ ${ }^{3}$ DFKI GmbH, Saarbrucken, Germany \\ florian.daiber@dfki.de, krueger@dfki.de \\ ${ }^{4}$ Department of Computer Science, University of Würzburg, Würzburg, Germany \\ frank.steinicke@uni-wuerzburg.de
}

\begin{abstract}
The penetration of stereoscopic 3D (S3D) output devices is becoming widespread. S3D screens range in size from large cinema screens, to tabletop displays, TVs in living rooms, and even mobile devices are nowadays equipped with autostereoscopic 3D screens. As a consequence, the requirement for interacting with 3D content is also increasing, with "touch" being one of the dominant input methods. However, the design requirements and best practices for interaction with S3D touch screen user interfaces are only now evolving. To understand the challenges and limitations S3D technology brings to interaction design, and in particular the additional demands they place on the users of such interfaces, we present our research that considers stereoscopic touch screens of different sizes in static and mobile usage contexts. We report on perceptual, cognitive and behavioral aspects revealed by user studies and present interaction design requirements based on the findings.
\end{abstract}

Keywords. Stereoscopy, 3D User Interfaces, Tabletops, Mobile User Interfaces, User Studies.

\section{$1 \quad$ Introduction}

Display and visualization technologies are advancing on various frontiers, and today we see both increasingly bigger and smaller screens with ever increasing resolutions. One of the emerging trends here is the use of stereoscopic 3D (S3D). Stereoscopic 3D is probably most familiar to large audiences from 3D movies, and 3DTVs and S3D mobile devices are already mass-market products. The penetration of S3D devices is constantly growing in numbers. Autostereoscopic 3D displays which require no special glasses to experience the 3D effect can be found e.g. in 3D cameras, mobile phones, tablets and game consoles such as the Nintendo 3DS. The S3D technologies create the illusion of depth, such that graphical elements are perceived to pop up from

adfa, p. 1, 2011.

(C) Springer-Verlag Berlin Heidelberg 2011 
or sink below the surface level of the screen. With negative disparity (or parallax), the UI elements appear to float in front of the screen, and with positive disparity (or parallax), the UI elements appears behind the screen level (see Fig. 1).

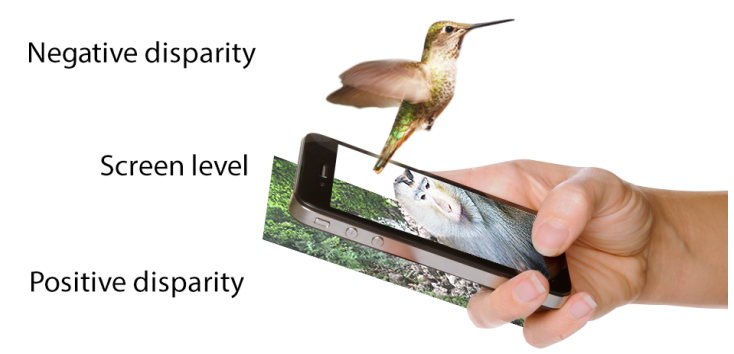

Fig. 1. An autostereoscopic 3D display, where the content is displayed with negative (bird), zero (monkey) and positive (background image) disparity / parallax.

So far, much of the research in the field of human-computer interaction (HCI) on S3D has focused on visual ergonomics and visual comfort (see e.g. [32], [33], [42]), and until now, research on interaction and user experience design for S3D has gained less attention. However, recent research has emerged in various application domains for $\mathrm{S} 3 \mathrm{D}$, ranging from interactive experiences in S3D cinemas [18], to mobile games [11], as well as in investigating the interaction design [6], [9], [51], [52].

The $3^{\text {rd }}$ dimension provides new degrees of freedom for designers, and the illusion of depth can be utilized both for information visualization as well as for interactive user interface (UI) elements. However, as the physical displays are still two-dimensional, it brings challenges on how to design interactive systems utilizing S3D. Especially, touch screen interaction is challenging with objects appearing visually in $3 \mathrm{D}$, but which you cannot physically touch. This mismatch between the visual and tangible perception can potentially increase the user's cognitive load, and make the systems slower to use and less easy to comprehend. Thus, it is important to investigate the effects that stereoscopic 3D can have on interaction compared to the conventional 2D UIs. In order to successfully introduce novel designs for S3D UIs, we need to investigate the implications of S3D on the user interaction e.g. in terms of touch accuracy and resolution.

In this paper, we investigate touch screen based interaction with $\mathrm{S} 3 \mathrm{D}$ visualizations, and compare the differences between S3D and 2D interaction. By examining the utility of S3D touchscreen UIs for practical usage when the user is interacting with different size S3D displays, we provide recommendations to researchers, developers and designers of such UIs. Especially, we:

- Provide a systematic analysis of the differences in interaction between 2D and S3D touch screens. 
- Investigate the touch screen based S3D interaction in the context of both large and small screens, namely tabletops and mobile devices

- Report on differences between S3D touch screen interaction while on the move (walking) and when stable (standing)

- Provide design recommendations for designing interactive touch S3D systems

\section{Related Work}

In this section we first briefly summarize the more general research on S3D in the area of $\mathrm{HCI}$ and than continue with a review of related work focusing on touch screen interaction with S3D.

\subsection{HCI Research in S3D.}

Visual Experience. With research on mobile S3D UIs, much of the emphasis so far has been focused on the output visualizations - video, images and visual cues - rather than on interacting with the device. Jumisko-Pyykkö et al. [28] studied the users' experience with mobile S3D videos, and discovered that the quality of experience was constructed from the following: visual quality, viewing experience, content, and quality of other modalities and their interactions. Pölönen et al. [42], report that with mobile S3D, the perceived depth is less sensitive to changes in the ambient illumination level than perceived naturalness and overall image quality. Mizobuchi et al. [37], report that S3D text legibility was better when text was presented at zero disparity on a positive disparity background image when compared to presenting it hovering on a background image that was at zero disparity. It has also been pointed out that scaling the S3D content to different size displays is not straightforward, and has an effect to the perceptual qualities [2].

Cognition and Perception. Considering the effect of S3D on users' cognition, Häkkinen et al. [21] have investigated people's focal attention with S3D movies using eye tracking. When comparing the S3D and 2D versions of the movies, they found that whereas in 2D movie viewers tend to focus quickly on the actors, in the S3D version the eye movements were more widely distributed among other targets. In their FrameBox and MirrorBox work, Broy et al. [5] identify that the number of depth layers has a significant effect on task completion time and recognition rate in a search task.

The role of visual cues has also been investigated, with some conflicting conclusions on their relative importance in depth perception. Mikkola et al. [36], concluding that the stereoscopic depth cues outperform the monocular ones in efficiency and accuracy, whilst Huhtala et al. [27] report that for a find-and-select task in a S3D mobile photo gallery, both the performance and subjective satisfaction were better when the stereoscopic effect was combined with another visual effect, i.e. dimming. Kerber et al. [30] investigated depth perception in a handheld stereoscopic augmented reality scenario. Their studies revealed that stereoscopy has a negligible if any effect on a small screen, even in favorable viewing conditions. Instead, the traditional depth 
cues, in particular object size, drive depth discrimination. The perception of stereoscopic depth on small screens has also been examined by Colley et al. [9], who compared users' ability to distinguish stereoscopic depth against their ability to distinguish 2D size. More recently, Mauderer et al. [35] investigated gaze- contingent depth of field as a method to produce realistic 3D images, and analyze how effectively people can use it to perceive depth.

Non-Touchscreen Interaction Techniques. Besides touchscreen based interaction, several other interaction methods have been applied to S3D interfaces. Teather et al. [49] demonstrated a "fish tank" virtual reality system for evaluating 3D selection techniques. Motivated by the successful application of Fitts' law to 2D pointing evaluation, the system provides a testbed for consistent evaluation of 3D point-selection techniques. The primary design consideration of the system was to enable direct and fair comparison between $2 \mathrm{D}$ and $3 \mathrm{D}$ pointing techniques. To this end, the system presents a 3D version of the ISO 9241-9 pointing task. Considering a layered stereoscopic UI, Posti et al. [41] studied the use of hand gestures to move objects between depth layers.

Gestures with mobile phones have also been utilized as an interaction technique. In the context of an S3D cinema Häkkilä et al. [18] utilized mobile phone gestures as one method to capture 3D objects from the film. Using a large-scale stereoscopic display Daiber et al. [10] investigated remote interaction with 3D content on pervasive displays, concluding that physical travel based techniques outperformed the virtual techniques.

Design Implications. The question of how the stereoscopic depth effect could be used in UI design has been investigated in several studies. Using S3D is perceived as a potential way to group similar content items or to highlight contextual information in a user interface [53]. There have also been design proposals where object depth within S3D UIs has been considered as an informative parameter e.g. to represent the time of the last phone call [20], and to identify a shared content layer in photo sharing [19]. Daiber et al. [11] investigated sensor-based interaction with stereoscopic displayed 3D data on mobile devices and presented a mobile 3D game that makes use of these concepts. Broy et al. have explored solutions for using S3D for in-car applications [4].

When evaluating the manufacturer's UI in an off-the-shelf S3D mobile phone, Sunnari et al. [47] found that the S3D design was seen as visually pleasant and entertaining, but lacking in usability, and the participants had difficulties seeing any practical benefit of using stereoscopy. In order to gain other than just hedonistic value, stereoscopy should be incorporated to the mobile UI in a way that it improves not only the visual design, but also the usability [17]. Considering this, more research on S3D for mobile devices equipped with an autostereoscopic display for both user experience and depth perception is still needed. 


\subsection{Touch Screen Interaction and S3D}

In the monoscopic case the mapping between an on-surface touch point and the intended object point in the virtual scene is straightforward, but with stereoscopic projection this mapping introduces problems [48]. To enable direct 3D "touch" selection of stereoscopically displayed objects in space, 3D tracking technologies can capture a user's hand or finger motions in front of the display surface. Hilliges et al. [25] investigated an extension of the interaction space beyond the touch surface. They tested two depth-sensing approaches to enrich multi-touch interaction on a tabletop setup. Although 3D mid-air touch provides an intuitive interaction technique, touching an intangible object, i.e., touching the void [8], leads to potential confusion and a significant number of overshoot errors. This is due to a combination of three factors: depth perception is less accurate in virtual scenes than in the real world, see e.g. [44], the introduced double vision, and also vergence-accommodation conflicts. Since there are different projections for each eye, the question arises: where do users touch the surface when they try to "touch" a stereoscopic object?

As described by Valkov et al. [51], for objects with negative parallax the user is limited to touch interaction on the area behind the object, since - without additional instrumentation - touch feedback is only provided at the surface. Therefore the user has to reach through the visual object to reach the touch surface with her finger. If the user reaches into an object while focusing on her finger, the stereoscopic effect for the object will be disturbed, since the user's eyes are not accommodated and converged on the projection screen's surface. Thus the left and right stereoscopic images of the object's projection would appear blurred and could not be merged anymore. However, focusing on the virtual object leads to a disturbance of the stereoscopic perception of the user's finger, since her eyes are converged to the object's $3 \mathrm{D}$ position. In both cases touching an object may become ambiguous [51]. To reduce the perception problems associated with reaching through an object to the touch screen surface, Valkov et al. [50] created a prototype where the selected object moved with the user's finger to the screen surface.

In principle, the user may touch anywhere on the surface to select a stereoscopically displayed object. However, in perceptual experiments Valkov et al. [51] found that users actually touch an intermediate point that is located between both projections with a significant offset towards the user's dominant eye. Bruder et al. [7] compared 2D touch and 3D mid-air selection in a Fitts' Law experiment for objects that are projected with varying disparity. Their results show that the $2 \mathrm{D}$ touch performs better close to the screen, while $3 \mathrm{D}$ selection outperforms $2 \mathrm{D}$ touch for targets further away from the screen.

Multi-touch technology provides a rich set of interactions without any instrumentation of the user but the interaction is often limited to almost zero disparity [43]. Recently, multi-touch devices have been used for controlling the 3D position of a cursor through multiple touch points [1], [46]. These can specify 3D axes or points for indirect object manipulation. Interaction with objects with negative parallax on a multitouch tabletop setup was addressed by Benko et al.'s balloon selection [1], as well as Strothoff et al.'s triangle cursor [46], which use 2D touch gestures to specify height 
above the surface. Grossman \& Wigdor [16] provided an extensive review of the existing work on interactive surfaces and developed a taxonomy for classification of this research.

Considering the mobile device domain, a comprehensive body of work exists examining the input accuracy of 2D touch screens, e.g. Holtz and Baudisch [26] and Parhi et al. [39]. Holtz's summary, that inaccuracy is largely due to a "parallax" artifact between user control based on the top of the finger and sensing based on the bottom side of the finger, is particularly relevant, as in the S3D case another on-screen parallax effect is introduced. On small mobile screens touch accuracy is even more critical than on large touch devices. The effect of walking on touch accuracy for $2 \mathrm{D}$ touch screen UIs has also been previously researched [3], [29], [40]. For example, Kane [29] finds variation in the optimal button size for different individuals, whilst Perry and Hourcade [40] conclude that walking slows down users' interaction but has little direct effect on their touch accuracy.

\subsection{Advancements over Related Work}

Taking the strong emphasis on visual design in S3D products and the output orientated prior art, there is a clear need to further investigate the interaction design aspects of S3D UIs. In this chapter, we focus on assessing touch screen interaction with S3D UIs in both large-screen tabletop and small screen mobile formats, in particular considering the target selection accuracy of objects at different depths and positions. Our aim is to provide practical information to assist the designers of S3D UIs.

Additionally, an understanding of the effect of the mobile domain on users' input accuracy when selecting targets is critical for the mobile UI designer. This extends the current body of research which has focused either to evaluate S3D in static conditions, or interaction with a 2D UI whilst on the move; the combination of S3D and user motion being relatively unstudied.

\section{Study I - Tabletop S3D Interaction}

In this section, we describe experiments in which we analyzed the touch behavior as well as the precision of $2 \mathrm{D}$ touching of $3 \mathrm{D}$ objects displayed stereoscopically on a tabletop surface. We used a standard ISO 9241-9 selection task setup on a tabletop surface with $3 \mathrm{D}$ targets displayed at different heights above the surface, i.e., with different negative parallaxes. Further details about this study and a comparison to 3D selection can be found in [7]. In this section, we focus on the S3D touch technique, in which subjects have to push their finger through the 3D stereoscopically displayed object (i.e. with negative parallax) until it reaches the $2 \mathrm{D}$ touch surface.

\subsection{Participants}

Ten male and five female subjects (ages 20-35, M=27.1, heights $158-193 \mathrm{~cm}$, $\mathrm{M}=178.3 \mathrm{~cm}$ ) participated in the experiment. Subjects were students or members of 
the Departments of computer science, media communication or human computerinteraction. Three subjects received class credit for participating in the experiment. All subjects were right-handed. We used the Porta and Dolman tests (see [31]), to determine the sighting dominant eye of subjects. This revealed eight right-eye dominant subjects ( 7 males, 1 female) and five left-eye dominant subjects ( 2 males, 3 females). The tests were inconclusive for two subjects ( 1 male, 1 female), for which the 2 tests indicated conflicting eye dominance. All subjects had normal or corrected to normal vision. One subject wore glasses and four subjects wore contact lenses during the experiment. None of the subjects reported known eye disorders, such as color weaknesses, amblyopia or known stereopsis disruptions. We measured the interpupillary distance (IPD) of each subject before the experiment, which revealed IPDs between $5.8 \mathrm{~cm}$ and $7.0 \mathrm{~cm}(\mathrm{M}=6.4 \mathrm{~cm})$. We used each individual's IPD for stereoscopic display in the experiment. Altogether 14 subjects reported experience with stereoscopic 3D cinema, 14 reported experience with touch screens, and 8 had previously participated in a study involving touch surfaces. Subjects were naive to the experimental conditions. Subjects were allowed to take a break at any time between experiment trials in order to minimize effects of exhaustion or lack of concentration. The total time per subject including pre-questionnaires, instructions, training, experiment, breaks, post-questionnaires, and debriefing was about 1 hour.

\subsection{Study Design}

Materials. For the experiment we used a $62 \times 112 \mathrm{~cm}$ multi-touch enabled active stereoscopic tabletop setup as described in [7]. The system uses rear diffuse illumination for multi-touch. For this, six high-power infrared (IR) LEDs illuminate the screen from behind. When an object, such as a finger or palm, comes in contact with the diffuse surface it reflects the IR light, which is then sensed by a camera. We use a $1024 \times 768$ PointGrey Dragonfly2 with a wide-angle lens and a matching IR band-pass filter at 30 frames per second.

We use a modified version of the NUI Group's CCV software to detect touch input on a Mac Mini server. Our setup uses a matte diffusing screen with a gain of 1.6 for the stereoscopic back projection. We used a 1280x800 Optoma GT720 projector with a wide-angle lens and an active DLP-based shutter at $60 \mathrm{~Hz}$ per eye. Subjects indicated target selection using a Razer Nostromo keypad with their non-dominant hand. To enable view-dependant rendering, an optical WorldViz PPT X4 system with submillimeter precision and sub-centimeter accuracy was used to track the subject's head in $3 \mathrm{D}$, based on wireless markers attached to the shutter glasses. Additionally, although not reported on in the scope of this chapter, a diffused IR LED on the tip of the index finger of the subject's dominant hand enabled tracking of the finger position in 3D (See Bruder et al. 2013).

The visual stimulus consisted of a $30 \mathrm{~cm}$ deep box that matches the horizontal dimensions of the tabletop setup (see Fig. 2). The targets in the experiment were represented by spheres, which were arranged in a circle, as illustrated in Figure 2. A circle consisted of 11 spheres rendered in white, with the active target sphere highlighted in blue. The targets highlighted in the order specified by ISO 9241-9. The center of each 
target sphere indicated the exact position where subjects were instructed to touch with their dominant hand in order to select a sphere. The size, distance, and height of target spheres were constant within circles, but varied between circles. Target height was measured as positive height from the screen surface. The virtual scene was rendered on an Intel Core i7 $3.40 \mathrm{GHz}$ computer with $8 \mathrm{~GB}$ of main memory, and an Nvidia Quadro 4000 graphics card.

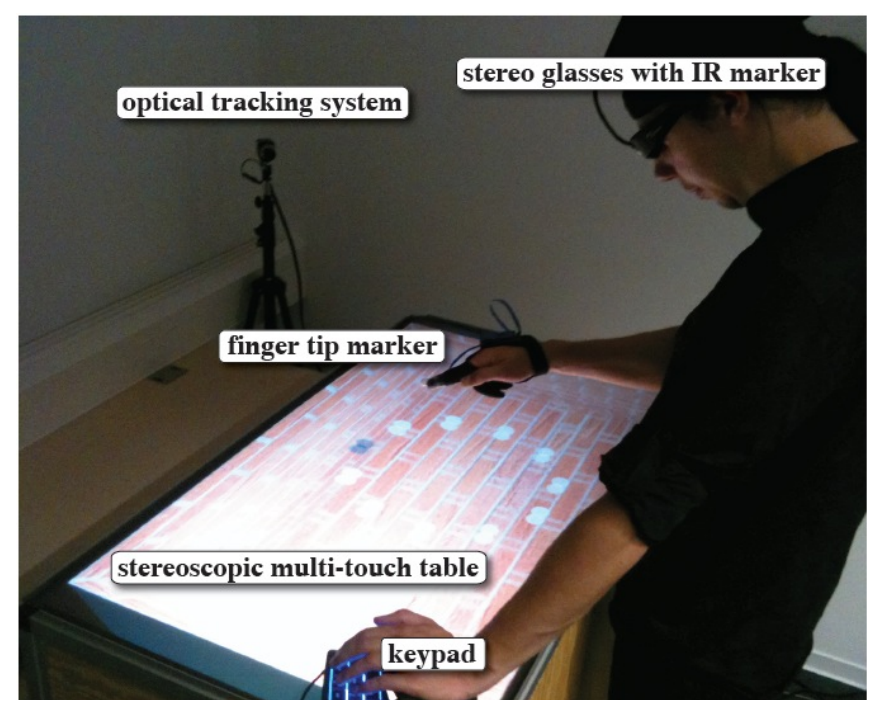

Fig. 2. Experiment setup: photo of a subject during the experiment (with illustrations). As illustrated on the screen, the target objects are arranged in a circle.

Test Procedure. For our experimental analyses and description we used a $5 \times 2 \times 2$ within-subjects design with the method of constant stimuli, in which the target positions and sizes are not related from one circle to the next, but presented randomly and uniformly distributed. The independent variables were target height (between $0 \mathrm{~cm}$ and $20 \mathrm{~cm}$, in steps of $5 \mathrm{~cm})$, as well as target distance $(16 \mathrm{~cm}$ and $25 \mathrm{~cm}$ ) and target size $(2 \mathrm{~cm}$ and $3 \mathrm{~cm})$. Each circle represented a different index of difficulty (ID), with combinations of 2 distances and 2 sizes. The ID indicates overall task difficulty [15]. It implies that the smaller and farther a target, the more difficult it is to select quickly and accurately. Our design thus uses four uniformly distributed IDs ranging from approximately $2.85 \mathrm{bps}$ to $3.75 \mathrm{bps}$, representing an ecologically valuable range of difficulties for such a touch-enabled stereoscopic tabletop setup. As dependent variables we measured the on-display touch areas for 3D target objects.

At the start of the test, subjects were positioned standing in an upright posture in front of the tabletop surface as illustrated in Figure 2. To improve comparability, we compensated for the different heights of the subjects by adjusting a floor mat below the subject's feet, resulting in an (approximately) uniform eye height of $1.85 \mathrm{~cm}$ for each subject during the experiment. The experiment started with task descriptions, which were presented via slides on the tabletop surface to reduce potential experi- 
menter bias. Subjects completed 5 to 15 training trials to ensure that they correctly understood the task and to minimize training effects. Training trials were excluded from the analysis.

In the experiment, subjects were instructed to touch the center of the target spheres as accurately as possible, for which they had as much time as needed. For this, subjects had to push their finger through the 3D sphere until it reached the $2 \mathrm{D}$ touch surface. Subjects did not receive feedback whether they "hit" their target, i.e., subjects were free to place their index finger in the real world where they perceived the virtual target to be. We did this to evaluate the often-reported systematical over- or underestimation of distances in virtual scenes, which can be observed even for short graspingrange distances, as also tested in this experiment. Moreover, we wanted to evaluate the impact of such misperceptions on touch behavior in stereoscopic tabletop setups. We tracked the tip of the index finger. When subjects wanted to register the selection, they had to press a button with their non-dominant hand on the keypad. We recorded a distinct 2D touch position for each target location for each configuration of independent variables.

\subsection{Results}

In this section we summarize the results from the tabletop S3D touch experiment. We had to exclude two subjects from the analysis who obviously misunderstood the task. We analyzed these results with a repeated measure ANOVA and Tukey multiple comparisons at the $5 \%$ significance level (with Bonferonni correction).

We evaluated the judged 2D touch points on the surface relative to the potential projected target points, i.e., the midpoint (M) between the projections for both eyes, as well as the projection for the dominant (D), and the non-dominant $(\mathrm{N})$ eye. Figure 3 shows scatter plots of the distribution of the touch points from all trials in relation to the projected target centers for the dominant and non-dominant eye for the different heights of $0 \mathrm{~cm}, 5 \mathrm{~cm}, 10 \mathrm{~cm}, 15 \mathrm{~cm}$ and $20 \mathrm{~cm}$ (bottom to top). We normalized the touch points in such a way that the dominant eye projection $\mathrm{D}$ is always shown on the left, and the non-dominant eye projection $\mathrm{N}$ is always shown on the right side of the plot. The touch points are displayed relatively to the distance between both projections.

As illustrated in Fig. 3, we observed three different behaviors. In particular, eight subjects touched towards the midpoint, i.e., the center between the dominant and nondominant eye projections. This includes the two subjects for whom eye dominance estimates were inconclusive. We arranged these subjects into the group $\mathrm{G}_{\mathrm{M}}$. Furthermore, three subjects touched towards the dominant eye projection $\mathrm{D}$, which we refer to as group $\mathrm{G}_{\mathrm{D}}$, and three subjects touched towards the non-dominant eye projection $\mathrm{N}$, which we refer to as group $\mathrm{G}_{\mathrm{N}}$. This points towards an approximately $50 / 50 \%$ split in terms of behaviors in the population, i.e., between group $\mathrm{G}_{\mathrm{M}}$ and the composite of groups $\mathrm{G}_{\mathrm{D}}$ and $\mathrm{G}_{\mathrm{N}}$.

We found a significant main effect of the three groups $(F(2,11)=71.267, p<.001$, partial-eta ${ }^{2}=.928$ ) on the on-surface touch areas. Furthermore, we found a significant two-way interaction effect of the three groups and target heights $(F(8,44)=45.251$, 
$\mathrm{p}<.001$, partial-eta ${ }^{2}=.892$ ) on the on-surface touch areas. The post-hoc test revealed that the on-surface target areas significantly vary $(\mathrm{p}<.001)$ for objects that are displayed at heights of $15 \mathrm{~cm}$ or higher. For objects displayed at $10 \mathrm{~cm}$ height group $\mathrm{G}_{\mathrm{D}}$ and $\mathrm{G}_{\mathrm{N}}$ vary significantly $(\mathrm{p}<.02)$. For objects displayed below $10 \mathrm{~cm}$ we could not find any significant difference. As illustrated in Figure 3, for these heights the projections for the dominant and non-dominant eye are close together, and subjects touched almost the same on-screen target areas.

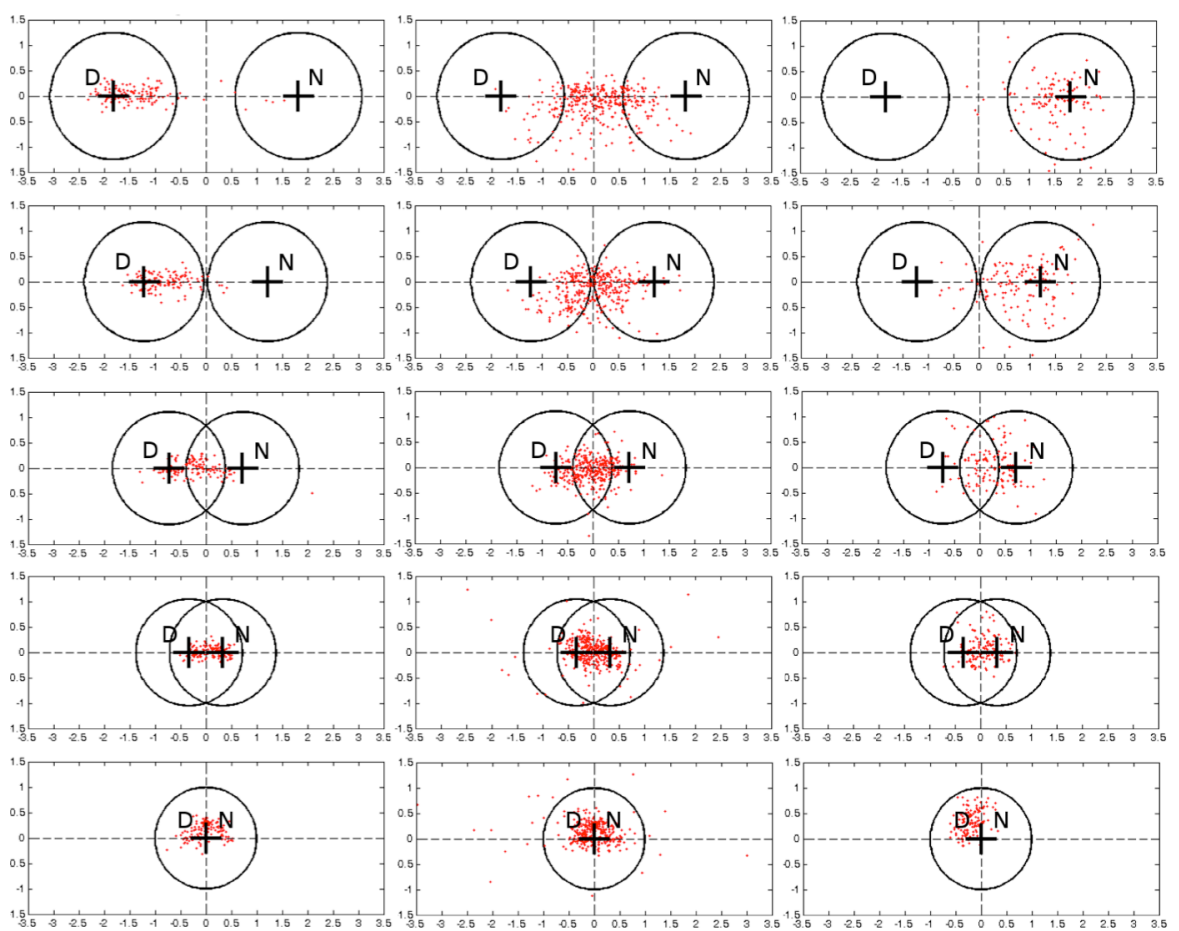

Fig. 3. Scatter plots of relative touch points between the dominant (D) and non-dominant (N) eye projections of the projected target centers on the surface for the 2D touch technique. Black crosses indicate the two projection centers. Black circles indicate the approximate projected target areas for the dominant and non-dominant eye. Top to bottom rows show results for $20 \mathrm{~cm}, 15 \mathrm{~cm}, 10 \mathrm{~cm}, 5 \mathrm{~cm}$, and $0 \mathrm{~cm}$ target heights. The left column shows subject behavior for dominant-eye touches ( 3 subjects), the middle for center-eye touches ( 8 subjects), and the right for non-dominant-eye touches ( 3 subjects). Note that the distance between the projection centers depends on the target height.

Considering the on-surface touch areas, we found that on average the relative touch point for group $G_{D}$ was $0.97 \mathrm{D}+0.03 \mathrm{~N}$ for projection points $\mathrm{D} \in \mathbb{R}^{2}$ and $\mathrm{N} \in \mathbb{R}^{2}$, meaning the subjects in this group touched towards the projection for the dominant eye, but slightly inwards to the center. The relative touch point for group $\mathrm{G}_{\mathrm{N}}$ was $0.11 \mathrm{D}+0.89 \mathrm{~N}$, meaning the subjects in this group touched towards the projection for the non-dominant eye, again with a slight offset towards the center. Finally, for group $\mathrm{G}_{\mathrm{M}}$ we found that on average the relative touch point for this group was 
$0.504 \mathrm{D}+0.596 \mathrm{~N}$. We could not find any significant difference for the different heights, i.e., the touch behaviors were consistent throughout the tested heights.

However, we observed a trend of target height on the standard deviations of the horizontal distributions ( $x$-axis) of touch points for all groups as shown in Figure 3. For $0 \mathrm{~cm}$ target height we found a mean standard deviation (SD) of $0.29 \mathrm{~cm}$, for $5 \mathrm{~cm}$ SD $0.32 \mathrm{~cm}$, for $10 \mathrm{~cm} \mathrm{SD} 0.42 \mathrm{~cm}$, for $15 \mathrm{~cm}$ SD $0.52 \mathrm{~cm}$, and for $20 \mathrm{~cm}$ SD $0.61 \mathrm{~cm}$. For the vertical distribution ( $y$-axis) of touch points and at $0 \mathrm{~cm}$ target height we found a mean SD of $0.20 \mathrm{~cm}$, for $5 \mathrm{~cm} \mathrm{SD} 0.20 \mathrm{~cm}$, for $10 \mathrm{~cm} \mathrm{SD} 0.25 \mathrm{~cm}$, for $15 \mathrm{~cm} \mathrm{SD}$ $0.29 \mathrm{~cm}$, and for $20 \mathrm{~cm} \mathrm{SD} 0.30 \mathrm{~cm}$.

Minimum Touch Target Sizes. For practical considerations and to evaluate the ecological validity of using the 2D touch technique for selections of targets at a height between $0 \mathrm{~cm}$ and $10 \mathrm{~cm}$, we computed the minimal on-surface touch area that supports $95 \%$ correct detection of all $2 \mathrm{D}$ touch points in our experiment. Due to the similar distributions of touch points between the three behavior groups for these heights shown in Figure 5, we determined the average minimal 95\% on-surface region over all participants. Our results show that an elliptical area with horizontal and vertical diameter of $1.64 \mathrm{~cm}$ and $1.07 \mathrm{~cm}$ with a center in the middle between the two projections is sufficient for $95 \%$ correct detection. This rule-of-thumb heuristic for onsurface target areas is easy to implement and ecologically valuable considering the fat finger problem [22], [34]. Due to this problem objects require a relatively large size of between $1.05 \mathrm{~cm}$ to $2.6 \mathrm{~cm}$ for reliable acquisition, even in monoscopic touch-enabled tabletop environments.

\section{$4 \quad$ Study II - Mobile S3D Interaction}

To systematically evaluate interaction with S3D in the mobile context, we designed a study to investigate users' performance when interacting with a mobile S3D device and compared this to the $2 \mathrm{D}$ case. Here, we examine interaction with both negative and positive parallax (compared to our tabletop study in which only on-screen and negative parallax were considered). In contrast to the tabletop study, the mobile study utilized an autostereoscopic display and hence did not require shutter glasses to be worn to see the stereoscopic effect. Additionally, in this study no viewer dependent adjustment of the rendered view was made. As mobility is by definition a core of this usage context, we also examined differences between interaction when static and walking.

\subsection{Participants}

The average age of the users ( $\mathrm{n}=27$ ) was 30.4 years (varying from 10 to 52 ), and 19 of the users were male and 8 were female. Tests to identify the user's dominant eye (Portas method, see [31]) and stereovision were conducted. Of the 27 users 16 were found to have a dominant right eye whilst 9 had a dominant left eye. Two users were unable to determine which of their eyes was dominant. 24 of the users were right- 
handed and the remaining 3 left-handed. Most had watched a 3D movie, and approximately half had tried out a $3 \mathrm{D}$ TV or a 3D camera.

\subsection{Study Design}

As we wanted to focus only on the depth effect due to the stereoscopy, no additional visual depth clues such as shadows, object size or color were used in our test application. In all the tests a background wallpaper image was used, as in pilot tests the use of some type of textured background at a positive disparity/parallax (Figure 1) was found to improve 3D perception. The background image chosen was a fine mesh pattern at $45^{\circ}$. This was chosen as it gave good $3 \mathrm{D}$ perception, but was such that it would not adversely influence the position of the users' presses on targets. In the 2D tests the background was positioned at $\mathrm{z}$-depth $=$ zero and in the $3 \mathrm{D}$ tests at $\mathrm{z}$-depth $=10$. Table 1 describes the z-depth convention and values used in the study. All the tests were conducted on an autostereoscopic touchscreen mobile device, the LG P920 Optimus 3D mobile phone. This has a 4.3" display with a resolution of 480x800 pixels (with $217 \mathrm{ppi}$ ) and runs Android 2.3.5.

Our target was to investigate the fundamental accuracy of users, hence our method avoided the use of any UI elements such as buttons, whose visuals could influence the position in which users tapped the screen. Thus, we followed a method similar to the crosshairs approach employed by Holtz and Baudisch [26]. Our accuracy test method presented rather small circular targets, one at a time. The user was instructed to tap on the center of each target with their index finger. For each tap the coordinates of both the press and release touch events were logged, as well as the time between the target being displayed and the user pressing the target.

The accuracy test was conducted in the 2D and S3D condition. In the 2D condition a random sequence of 15 targets was presented. Each target was presented once and once only. The targets were positioned in a grid pattern of 5 horizontal by 3 vertical. This pattern was chosen to give more data points and resolution in the horizontal axis, which is the most interesting for S3D. The targets and background image were displayed as normal $2 \mathrm{D}$ objects.

Table 1. Reference stereoscopic depths and calculated apparent distance behind the display (assuming viewing distance of $330 \mathrm{~mm}$ and inter-pupil distance of $63 \mathrm{~mm}$ )

\begin{tabular}{ll}
\hline Accuracy test z-depths & Apparent distance behind screen $(\mathrm{mm})$ \\
\hline 10 & 12.0 \\
3 & 3.6 \\
2 & 2.5 \\
0 & 0 (on screen) \\
-1 & -1.2 (in front of screen) \\
-2 & -2.5 (in front of screen) \\
\hline
\end{tabular}

In the S3D version of the accuracy test the same grid of 15 targets as used in the 2D test was used, but it was positioned at 5 different $\mathrm{z}$-depths (see Table 1), thus mak- 
ing a total of 75 targets. The 75 targets were presented in a random order of $\mathrm{x}, \mathrm{y}$ and $\mathrm{z}$ position, such that each target was presented once and once only. In the S3D test the background wallpaper image was positioned at depth $z=10$, behind the screen. It should be noted that the due to the difference in background depth the S3D test with targets at $\mathrm{z}=0$ was not exactly equivalent to the $2 \mathrm{D}$ test.

Based on an eyes-to-screen distance of $330 \mathrm{~mm}$ (the mean of 3 pilot users) and an inter-pupil distance of $63 \mathrm{~mm}$ (see [14]) the object distances in front of and behind the screen are given in Table 1. These values give an approximation of the apparent depth of objects presented at the different reference depths (the distance of the screen from the eyes was not fixed during the tests).

\subsection{Test Procedure}

An acclimatization task of looking through an S3D image gallery, provided as default by the device manufacturer, enabled users to get accustomed to the S3D effect. Users were instructed to experiment to find the best position, i.e. distance from device to eyes, viewing angle etc. where they could best see the 3D effect of the gallery images.

Each of the interaction tests: 2D accuracy and S3D accuracy were conducted in two different conditions; while seated (static) and whilst walking (on the move). For the walking tests, a route consisting of two markers $6 \mathrm{~m}$ apart was marked on the laboratory floor. Users were instructed to walk at normal speed around the marked route whilst completing the tests. The presentation order of the conditions was counterbalanced between participants, to reduce any effect of user learning or other side effects.

\subsection{Results}

Analysis of the accuracy tests is based on the recorded co-ordinates of the touch press event (i.e. "finger down"), as this defines the users' fundamental accuracy. Presses that were more than $12 \mathrm{~mm}$ from the center of the visual target were considered as accidental and excluded from further analysis (Removed data: static $2 \mathrm{D}=2,3 \mathrm{D}=4$, walking $2 \mathrm{D}=3,3 \mathrm{D}=10$ ).

Heatmaps of the press points for each test are shown in Figure 4., which combines the presses for all of the depth layers. These charts plot the offset of each press point from the center of the presented circular target, i.e. the center of the visual target is at the origin of the chart.

The absolute distance of each press point from the center of the visual target (i.e. ignoring the direction of the error) is presented in Fig. 5. For the S3D layers (i.e. within the groups of five bars in Fig. 5), there was no significant difference between the mean error distance for each of the 3D depth layers (based on ANOVA analysis). Thus, targets at screen level $(\mathrm{z}=0)$ were pressed no more accurately than targets at other depths. For all the S3D depth layers combined together, a 2-way ANOVA test revealed both walking and $\mathrm{S} 3 \mathrm{D}$ caused a significant increase in the mean error distance, $(\mathrm{F}(2,4841)=28.8, \mathrm{p}<.001)$ and $(\mathrm{F}(2,4841)=54.9, \mathrm{p}<.001)$, respectively. There was no significant interaction between the fixed factors. 

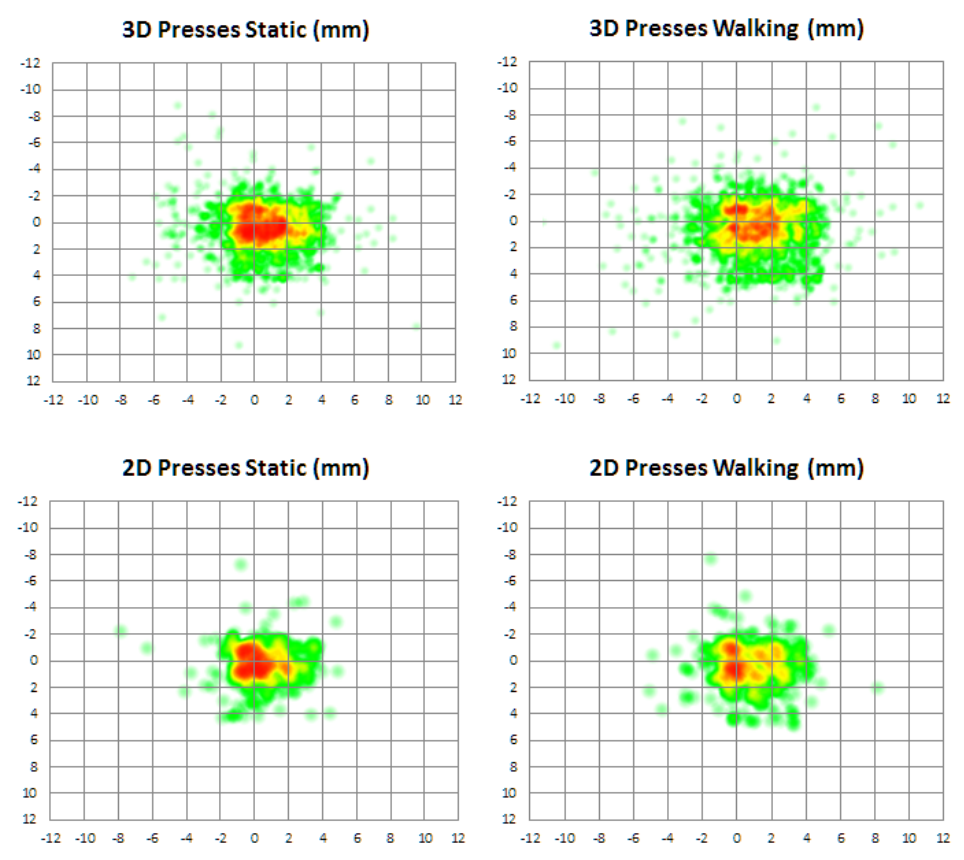

Fig. 4. Combined press points relative to target center for S3D and 2D.

Comparing the $\mathrm{S} 3 \mathrm{D} \mathrm{z}=0$ results, where the target is placed at screen depth, with the corresponding 2D cases, where the target is also at screen depth (circled on Fig. 5), showed significant degradation in accuracy in the S3D cases in comparison to 2D cases for $\mathrm{z}=0(\mathrm{~T}(403)=3.52, \mathrm{p}<.001$ and $\mathrm{T}(402)=4.35, \mathrm{p}<.001$ for static and walking cases respectively). This difference is due to the $\mathrm{S} 3 \mathrm{D}$ cases having a background image at positive disparity, placing the targets within a stereoscopic scene.

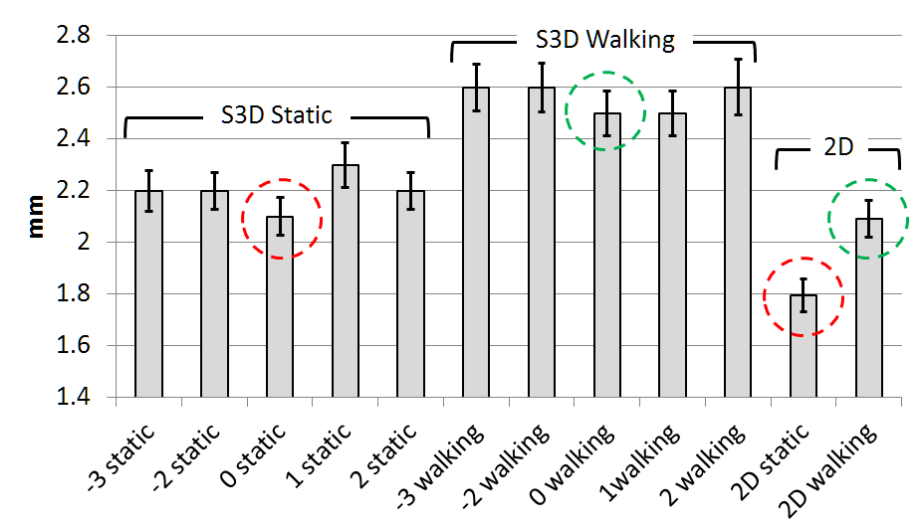

Fig. 5. Mean distance from target center for 2D \& individual 3D layers whilst static and walking. Error bars show standard error of mean. 
Additionally, in the $\mathrm{S} 3 \mathrm{D}$ cases, the $\mathrm{z}=0$ targets are presented in a sequence of targets at other depths. These findings are important as they indicate that from the touch accuracy point of view, there is no benefit to place targets at screen depth compared to any other depth level. Rather, to maximize touch accuracy, it is recommended that if $3 \mathrm{D}$ is not beneficial for other purposes within a UI, it is turned off.

Following a similar method to that employed in Study I, we separated users based on their dominant eyes. However in this case we were unable to ascertain any significance between the groups.

Minimum Touch Target Sizes. One approach to evaluate the degradation in accuracy caused by walking and $\mathrm{S} 3 \mathrm{D}$ is to calculate the minimum touch target sizes for each case. The minimum touch target size is the size required for users' to reliably hit touch targets in each tested mode. From the press point error distribution we calculated the 98 percentile points in positive and negative directions for both $\mathrm{x}$ and $\mathrm{y}$. Taking the approach described by [39], this defines the bounding rectangle that captures $95 \%$ of user presses. As the distributions are slightly skewed to either positive or negative sides, and in practice it is not possible to position a touch target at the exactly the correct offset from the center, thus we take double the larger absolute value, which may be considered as the minimum size for targets in the UI. It should be noted that these values are based on index finger usage, as in the test protocol. For thumb usage sizes will be somewhat larger (see [39]). The minimum target sizes are shown in Table 2. and diagrammatically in Fig. 6.

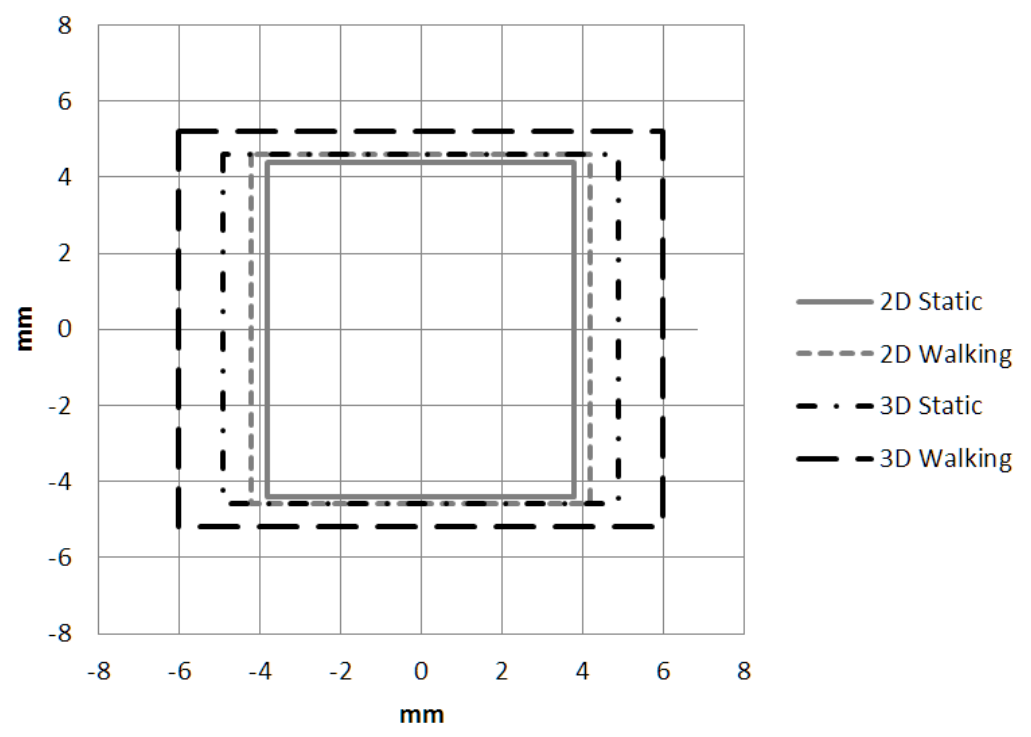

Fig. 6. Minimum target sizes for finger usage (95\% press success) 
Table 2. Minimum target sizes for finger usage.

\begin{tabular}{lccc}
\hline Conditions & $\begin{array}{l}\text { Negative } \\
98 \text { percentile } \\
(\mathrm{mm})\end{array}$ & $\begin{array}{l}\text { Positive } \\
98 \text { percentile } \\
(\mathrm{mm})\end{array}$ & $\begin{array}{l}\text { Minimum target size } \\
\text { (95\% presses on target) } \\
\text { width } \mathrm{x} \text { height }(\mathrm{mm})\end{array}$ \\
\hline 2D Static & $\mathrm{x}: 3.5$ & $\mathrm{x}: 3.8$ & $7.6 \times 8.8$ \\
& $\mathrm{y}: 3.9$ & $\mathrm{y}: 4.4$ & \\
2D Walking & $\mathrm{x}: 3.6$ & $\mathrm{y}: 4.2$ & \\
& $\mathrm{y}: 3.7$ & $\mathrm{x}: 4.6$ & \\
3D Static & $\mathrm{x}: 4.9$ & $\mathrm{y}: 4.6$ & $9.8 \times 9.2$ \\
& $\mathrm{y}: 3.8$ & $\mathrm{x}: 5.0$ & $12.0 \times 10.4$ \\
3D Walking & $\mathrm{x}: 6.0$ & $\mathrm{y}: 5.2$ & \\
\hline
\end{tabular}

\section{Discussion}

\subsection{Touch Performance}

Examining our results for the tabletop case, the S3D touch technique shows a significant effect for the different user groups on the on-surface touch area over the range of tested heights. These on-surface touch areas vary significantly for objects displayed at heights of $10 \mathrm{~cm}$ and higher. In contrast to previous work by Valkov et al. [51, 52], our results show evidence for a twofold diversity of 2D touch behaviors of users. As shown in Fig. 3., roughly half of the subjects in our study touched through the virtual object towards the center between the projections, and the other half touched towards projections determined by a single eye. The second group roughly splits in half again depending if they touch the projection for the dominant or non-dominant eye.

In the mobile case, and in the tabletop case for targets closer to the screen than $10 \mathrm{~cm}$, we were not able to identify any significant effect based on the user's dominant eye. Hence concluding that this phenomenon is not relevant to targets that are close to the screen level. Further, in the case of the small screen, interaction issues related to the general touch interaction accuracy dominate.

\subsection{Minimum Touch Area}

For tabletop interaction, we determined the minimum on-surface touch area $(95 \%$ correct detection) for interacting with $3 \mathrm{D}$ targets within $10 \mathrm{~cm}$ of the screen surface, to be an elliptical area with horizontal and vertical diameter of $16.4 \mathrm{~mm}$ and $10.7 \mathrm{~mm}$ with a center in the middle between the two projections. For interaction with the mobile device the corresponding area was a slightly smaller rectangle with dimensions of $9.8 \mathrm{~mm} \times 9.2 \mathrm{~mm}$ when static, increasing to $12.0 \mathrm{~mm} \times 10.4 \mathrm{~mm}$ when walking. How- 
ever, further analysis of the differences is not informative, due to the large difference in depth display capabilities between the mobile device and tabletop $(1.2 \mathrm{~mm}$ vs. $10 \mathrm{~cm}$ and above).

Considering also 2D performance in the mobile case, and comparing with previous studies, we find close agreement. For example, using their contact area model Holtz and Baudisch [26] report a $7.9 \mathrm{~mm}$ target size for $95 \%$ accuracy, compared to our $7.6 \mathrm{~mm} \times 8.8 \mathrm{~mm}$ (height $\mathrm{x}$ width) for the same accuracy. The aspect ratio information in our results provides interesting additional detail.

\subsection{Mobile Context}

Direct comparison between our results related to the differences in touch target sizes when walking with prior work on this topic is not straightforward, due to differences in the study approaches used. The majority of previous work being based on a UI with varied size button targets, and measuring task completion time and error rates (for example [29]). In contrast, our button free approach aimed to minimize the influence of the visual UI design, and focused to the accuracy of separate tap events. Thus, in our test, accuracy was the only dependent variable.

Our approach enabled us to investigate interaction over the full screen area, and we were able to gain insight into the optimal aspect ratio of touch targets. However, our method is more abstract than actual UI based approaches, requires some interpretation to transfer it to use in actual designs, and does not take account of other interaction issues e.g. related to Fitts' law [15]. Hence, it is perhaps the case that our method serves best as an initial method, the results of which could be validated by a button and task based method.

Based on our results, for a certain screen size, a S3D UI for use whilst walking should have only $60 \%$ of the touch targets of a correspondingly performing $2 \mathrm{D}$ UI (based on area calculations from Table 2.). This is a major impact to the design of S3D mobile UIs. The degradation in accuracy from the combined effects of S3D and walking appears to be larger than the sum of each individually (Fig. 6). As may be expected the main part of this degradation is in the horizontal dimension, and hence related to stereoscopic effects. We speculate that, at least in part, this effect is due to users losing the stereoscopic effect, for example when glancing from the display when walking, which is an expected condition for real world interaction in mobile context (see [38]).

\subsection{Cognitive Issues of S3D Interaction}

Both tabletop and mobile studies highlighted a large variation in the performance of individuals, which, in the mobile case, was further exaggerated when the users were walking. This large variation makes it difficult to identify statistically significant generic differences between the cases examined without a very large test sample. When designing any user interface it is not wise to design for the average user, without considering the variation of user performance. In the case of S3D UIs this suggests that the use of depth as a sole informative element in the UI should be approached cau- 
tiously. Clearly, when accessibility for users with restricted capabilities is a consideration, the use of S3D, at least as an informative channel should be avoided.

\subsection{Future Work}

Interestingly, visual examination of the distribution of presses in the mobile case (see Fig. 6) suggests a slight shift to the right whilst walking. This appears similar for both 2D and S3D cases. The reason for this is unknown and would require further study. Possible causes could be differences in the distance between the users' eyes and the screen, or differences in the viewing angle. Such small differences may prove relevant for tasks requiring the pressing of very small targets, such as on screen QWERTY keyboards used for text input, whilst walking. These aspects require further study.

\section{Conclusions}

In this chapter we reported on the evaluation of 2D touch compared to 3D touch interaction techniques for scenes on touch-sensitive tabletops and touchscreen mobile devices with stereoscopic displays. We analyzed a technique based on reducing the 3D touch problem to two dimensions by having users touch "through" the stereoscopic impression of $3 \mathrm{D}$ objects, resulting in a $2 \mathrm{D}$ touch on the display surface.

Tabletop. In the case of tabletops, where the scale is relatively large, we identified two separate classes of user behavior, with one group that touches the center between the projections, whereas the other touches the projection for the dominant or nondominant eye. The results show a strong interaction effect between input technique and the stereoscopic parallax of virtual objects.

Mobile Device. For the smaller mobile device S3D touch screen, the mean press positions on visual targets at all z-depths were almost identical, i.e. there was no discernible offset caused by placing targets at different z-depths. However, the variance in press accuracy in the S3D case was much larger than for 2D, and this difference was more pronounced when walking. When in static usage, to achieve the same performance as $2 \mathrm{D}, \mathrm{S} 3 \mathrm{D}$ touch targets need to be horizontally wider $(7.6 \mathrm{~mm}$ for $2 \mathrm{D}$ vs. $9.8 \mathrm{~mm}$, for $3 \mathrm{D}$ for $95 \%$ presses on target).

To support usage whilst walking, the size of the touch targets on a touch screen S3D user interface needs to be significantly larger than the corresponding minimum for a $2 \mathrm{D}$ interface. In our study the minimum sizes for $95 \%$ presses on target was $8.4 \mathrm{~mm} \times 9.2 \mathrm{~mm}$ (width $\mathrm{x}$ height) for $2 \mathrm{D}$ vs. $12.0 \mathrm{~mm} \times 10.4 \mathrm{~mm}$ for 3D. Although these values are based on the test device used, it is expected that they should be generally applicable to other similar S3D devices. For example, the presented minimum touch target sizes in millimeters should serve as initial guidelines for UI designers of other S3D touch screen products intended for mobile use.

Contribution. The main contributions of this work are: 
- We have presented the minimum target size for S3D touch targets for both large screen tabletop and small screen mobile touch screen interaction.

- We identified two separate classes of user behavior when touching "through" stereoscopically displayed objects, on larger scale S3D displays (e.g. tabletops).

- We validated that the 2D touch technique performs well for selection of objects up to about $10 \mathrm{~cm}$ height from the display surface.

- For the mobile device case, both walking and S3D caused a significant increase in the mean error distance.

\section{References}

1. Benko, H. and Feiner, S. Balloon selection: A multi-finger technique for accurate lowfatigue 3D selection. Proc. IEEE 3DUI, pp. 79-86, (2007).

2. Benzeroual, K., Allison, R. S., Wilcox, L. M. 3D Display size matters: Compensating for the perceptual effects of S3D display scaling. In Proc. CVPRW 2012, 45-52. IEEE (2012).

3. Bergstrom-Lehtovirta, J., Oulasvirta, A., Brewster, S.: The effects of walking speed on target acquisition on a touchscreen interface. In Proc. MobileHCI 2011, ACM (2011).

4. Broy, N., André, E., \& Schmidt, A.: Is stereoscopic 3D a better choice for information representation in the car? In Proceedings of the 4th International Conference on Automotive User Interfaces and Interactive Vehicular Applications, pp. 93-100. ACM (2012).

5. Broy, N., Schneegass, S., Alt, F., \& Schmidt, A.: Framebox and mirrorbox: Tools and guidelines to support designers in prototyping interfaces for 3D displays. In proc. CHI'14 pp. 2037-2046. ACM (2014).

6. Bruder G., Steinicke F., Stuerzlinger W.: Effects of Visual Conflicts on 3D Selection Task Performance in Stereoscopic Display Environments. In proc. 3DUI'10, pp.115-118, IEEE (2013).

7. Bruder, G.; Steinicke, F., Sturzlinger, W.: To Touch or Not to Touch?: Comparing 2D Touch and 3D Mid-air Interaction on Stereoscopic Tabletop Surfaces. In Proc. SUI 2013. ACM (2013).

8. Chan, L. W., Kao, H. S., Chen, M. Y., Lee, M. S., Hsu, J., \& Hung, Y. P.: Touching the void: direct-touch interaction for intangible displays. In Proc. CHI'10 pp. 2625-2634. ACM (2010).

9. Colley, A., Häkkilä, J., Schöning, J., Posti, M.: Investigating Mobile Stereoscopic 3D Touchscreen Interaction. In Proc. OzCHI'13. ACM (2013).

10. Daiber, F., Speicher, M., Gehring, S., Löchtefeld, M., Krüger, A.: Interacting with 3D Content on Stereoscopic Displays. In proc. PerDis'14. ACM (2014).

11. Daiber, F., Li, L., Krüger, A.: Designing Gestures for Mobile 3D Gaming, In Proc. MUM'12, ACM (2012).

12. Daiber, F., Falk, E. \& Krüger, A.: Balloon Selection revisited: Multi-touch Selection Techniques for Stereoscopic Data. In Proc. AVI 2012, ACM, 441-444 (2012).

13. de la Riviere J.-B., Kervegant C., Orvain E., and Dittlo N.: Cubtile: a multi-touch cubic interface. Proc. ACM VRST, pp. 69-72. ACM (2008).

14. Dodgson, Neil A.: Variation and extrema of human interpupillary distance. Stereoscopic Displays and Applications. In Proc. SPIE 2004, 5291: 36-46. (2004).

15. Fitts, P. M.: The information capacity of the human motor system in controlling the amplitude of movement, Journal of Experimental Psychology, 47, pp. 381-391. (1954). 
16. Grossman, T., \& Wigdor, D.: Going Deeper: a Taxonomy of 3D on the Tabletop. In Horizontal Interactive Human-Computer Systems, 2007. TABLETOP'07. Second Annual IEEE International Workshop on (pp. 137-144). IEEE (2007).

17. Häkkilä, J., Kytökorpi, K., Karukka M.: Mobile Stereoscopic 3D User Experience - Calling for User Centric Design. In Proc. 3DCHI workshop at CHI'12. (2012).

18. Häkkilä, J., Posti, M., Schneegass, S., Alt, F., Gultekin, K., Schmidt, A.: Let me catch this! Experiencing Interactive 3D Cinema through Collecting Content with a Mobile Phone. In Proc. CHI 2014. ACM (2014).

19. Häkkilä, J., Posti, M., Ventä-Olkkonen, L., Koskenranta, O., Colley, A.: Mobile Photo Sharing through Collaborative Space in Stereoscopic 3D. In Proc. MUM 2013. ACM (2013).

20. Häkkilä, J., Posti, M., Koskenranta, O., \& Ventä-Olkkonen, L.: Design and evaluation of mobile phonebook application with stereoscopic 3D user interface. In CHI'13 Extended Abstracts on Human Factors in Computing Systems (pp. 1389-1394). ACM (2013).

21. Häkkinen, J., Kawaid, T., Takatalo, J., Mitsuya, R. and Nyman, G.: What do people look at when they watch stereoscopic movies? In Proc. SPIE'10 7524, (2010).

22. Hall, A. D., Cunningham, J. B., Roache, R. P. And Cox, J. W.: Factors affecting performance using touch entry systems: Tactual recognition fields and system accuracy. In J. Appl. Psychol., 4:711-720, (1988).

23. Hassenzahl, M.: User Experience (UX): Towards an experiential perspective on product quality. In Proc. IMH 2008, ACM, 11-15. (2008).

24. Henze, N., Rukzio, E. and Boll, S.: 100,000,000 Taps: Analysis and Improvement of Touch Performance in the Large, In Proc. MobileHCI 2011. ACM (2011).

25. Hilliges O., Izadi S., Wilson A. D., Hodges S., Garcia-Mendoza A., and Butz A.: Interactions in the air: Adding further depth to interactive tabletops. Proc. ACM UIST'09, pp. 139-148, (2009).

26. Holtz, C. and Baudisch, P.: Understanding touch. In Proc. CHI'11, pp. 2501-2510. ACM (2011)

27. Huhtala, J., Karukka, M., Salmimaa M. and Häkkilä, J.: Evaluating Depth Illusion as Method of Adding Emphasis in Autostereoscopic Mobile Displays. In Proc. MobileHCI 2011. ACM (2011).

28. Jumisko-Pyykkö, S., Dominik Strohmeier, D., Utriainen, T., Kunze, K.: Descriptive Quality of Experience for Mobile 3D Video. In Proc. NordiCHI, 2010, 266-275. ACM (2010).

29. Kane, S., Wobbrock, J., Smith, I.: Getting off the treadmill: evaluating walking user interfaces for mobile devices in public spaces. In Proc. MHCI08, 109-118. (2008).

30. Kerber, F., Lessel, P., Mauderer, M., Daiber, F., Oulasvirta, A., Krüger, A.: Is Autostereoscopy Useful for Handheld AR? In Proc. MUM'13, ACM (2013).

31. Kommerell G, Schmitt C, Kromeier M, Bach M.: Ocular prevalence versus ocular dominance. Vision Res. 2003; 43:1397-1403. (2003)

32. Kooi, F., Toet, A.: Visual Comfort of Binocular and 3-D Displays. Displays 25, 2004, 99 108. (2004).

33. Lambooij, M. T. M., Wijnand A. IJsselsteijn, W. A., Heynderickx, I.: Visual Discomfort in Stereoscopic Displays: A Review. Stereoscopic Displays and Virtual Reality Systems XIV, SPIE-IS\&T, (2007).

34. Loomis, J. M. and Knapp, J. M.: Visual perception of egocentric distance in real and virtual environments. In L. J. Hettinger and M. W. Haas, editors, Virtual and adaptive environments, volume Virtual and adaptive environments. Mahwah, (2003).

35. Mauderer, M., Conte, S., Nacenta, M. A., \& Vishwanath, D.: Depth perception with gazecontingent depth of field. In proc. CHI'14 pp. 217-226. ACM (2014). 
36. Mikkola, M., Boev, A., Gotchev, A.: Relative Importance of Depth Cues on Portable Autostereoscopic Display. In Proc. MoViD at ACM Multimedia 2010, 63-68. (2010).

37. Mizobuchi, S. et al.: The Effect of Stereoscopic Viewing in a Word Search Task with a Layered Background. Journal of the Society for Information Display 16/11, 1105-1113. (2008).

38. Oulasvirta, A., Tamminen, S., Roto, V., Kuorelahti, J.: Interaction in 4-second bursts: the fragmented nature of attentional resources in mobile HCI. In Proc. CHI '05. ACM (2005).

39. Parhi, P., Karlson, A. K., and Bederson, B. B.: Target size study for one-handed thumb use on small touchscreen devices. In Proc. MobileHCI 2006, ACM (2006).

40. Perry, K. and Hourcade, J. P.: Evaluating One Handed Thumb Tapping on Mobile Touchscreen Devices. In Proc. Graphics Interface, (2008). p. 57-64. (2008).

41. Posti, M., Ventä-Olkkonen, L., Colley, A., Koskenranta, O., Häkkilä, J.: Exploring Gesture Based Interaction with a Layered Stereoscopic 3D Interface. In Proc. PerDis'14. ACM (2014).

42. Pölönen, M., Salmimaa, M., Häkkinen, J. Effect of ambient illumination level on perceived autostereoscopic display quality and depth perception. Displays 32, 135-141. Elsevier (2011).

43. Schöning, J., Steinicke, F., Valkov, D., Krüger, A., Hinrichs, K. H.: Bimanual Interaction with Interscopic Multi-Touch Surfaces, In Proc. INTERACT '09. Springer (2009).

44. Sivak, B. and MacKenzie, C. L.: Integration of visual information and motor output in reaching and grasping: the contributions of peripheral and central vision. Neuropsychologia, 28(10):1095-1116. (1990).

45. Steinicke, F., Hinrichs, K. H., Schöning, J., Krüger, A.: Multi-Touching 3D Data: Towards Direct Interaction in Stereoscopic Display Environments coupled with Mobile Devices, In Proc. AVI Workshop on Designing Multi-Touch Interaction Techniques for Coupled Public and Private Displays '08, 46-49. (2008).

46. Strothoff S., Valkov D., and Hinrichs K. H.: Triangle cursor: Interactions with objects above the tabletop. In proc. ACM ITS '11, pp. 111-119. (2011).

47. Sunnari, M., Arhippainen, L., Pakanen, M., Hickey, S.: Studying User Experiences of Autostereoscopic 3D Menu on Touch Screen Mobile Device. In Proc. OZCHI'12, ACM (2012).

48. Teather, R. J., Stuerzlinger, W.: Pointing at 3D targets in a stereo head-tracked virtual environment, In Proc. 3DUI 2011, IEEE Computer Society, 87-94. (2011).

49. Teather, R. J., Stuerzlinger, W., Pavlovych, A.: Fishtank fitts: a desktop VR testbed for evaluating 3D pointing techniques. In CHI'14 Extended Abstracts on Human Factors in Computing Systems (pp. 519-522). ACM (2014).

50. Valkov, D., Giesler, A., Hinrichs, K. H.: Imperceptible depth shifts for touch interaction with stereoscopic objects. In Proceedings of the 32nd annual ACM conference on Human factors in computing systems (pp. 227-236). ACM (2014).

51. Valkov, D., Steinicke, F., Bruder, G., Hinrichs, K.: 2D Touching of 3D stereoscopic objects. In Proc. of CHI'11, ACM, 1353-1362. (2011).

52. Valkov, D., Steinicke, F., Bruder, G., Hinrichs, K., Schöning, J., Daiber, F., Krüger, A.: Touching Floating Objects in Projection-based Virtual Reality Environments. In Proc. EGVE/EuroVR/VEC 2010, 17-24. (2010).

53. Ventä-Olkkonen, L., Posti, M., Häkkilä, J. How to Use 3D in Stereoscopic Mobile User Interfaces - Study of Initial User Perceptions. In Proc. Academic MindTrek'13. ACM (2013). 